\title{
SPECTRA OF M DWARFS - DIMMED BY DUST
}

\author{
T. TSUJI, K. OHNAKA, W. AOKI AND H. R. A. JONES \\ Institute of Astronomy, The University of Tokyo \\ 2-21-1 Osawa, Mitaka, Tokyo, 181 Japan
}

\section{Introduction}

Spectra of $\mathrm{M}$ dwarfs are rich in atomic and molecular lines. These spectra provide such basic information as $T_{\text {eff }}$ (or radius), $\log g$ (or mass), surface chemical composition, and something more (e.g. activity) if properly interpreted. It is recognized, however, that spectra of $M$ dwarfs are already dimmed by the dust formed in their photospheres (Tsuji et al. 1996a) and this effect, which has been overlooked until recently, should be taken into account in any interpretation and analysis of the spectra of very low mass objects (VLMOs) including late M dwarfs and brown dwarfs.

\section{Dusty Model Atmospheres of M Dwarfs and Brown Dwarfs}

In cool and dense photospheres of VLMOs, the thermodynamical condition for condensation is well fulfilled. We first assume that the dust forms in the local thermodynamical equilibrium (LTE): This assumption is of course not trivial and we will return to this point later (Sect.4). Then, we consider condensation of such grains as $\mathrm{Al}_{2} \mathrm{O}_{3}$ (corundum), $\mathrm{Fe}$ (iron), and $\mathrm{MgSiO}_{3}$ (enstatite) formed from the most abundant refractory elements ( $\mathrm{Fe}, \mathrm{Mg}, \mathrm{Si}, \mathrm{Al}$ ). These condensates are effective sources of opacity due to their large extinction coefficients, which can be evaluated by the Mie theory (we assumed grain radius of $0.1 \mu \mathrm{m}$ ). The volatile molecules such as $\mathrm{CH}_{4}, \mathrm{H}_{2} \mathrm{O}, \mathrm{NH}_{3}, \mathrm{H}_{2} \mathrm{~S}, \mathrm{PH}_{3}$ remain as important sources of opacity, together with the collision-induced dipole transitions of $\mathrm{H}_{2}$.

Then, we constructed non-grey dusty model in radiative-convective equilibrium within the framework of the classical assumptions, and extended our grid of model atmospheres to $T_{\text {eff }}=$ $800 \mathrm{~K}$ (solar composition, $\log g=5.0$, and $v_{\text {micro }}=1 \mathrm{~km} \mathrm{~s}^{-1}$ ). The effects of dust appear in models of $T_{\text {eff }}=2,800 \mathrm{~K}$ and lower: dust (especially iron) effectively blocks the radiation and the photosphere is heated appreciably. For this reason, molecular bands such as $\mathrm{H}_{2} \mathrm{O}$ are much weaker than in dust-free model of the same $T_{\text {eff }}$. Also, optical spectra suffer large extinction by the dust.

\section{Evidences for Dust in Obseved Spectra and Colours}

We have already shown evidence for dust in late $M$ dwarfs (T'suji et al. 1996a), but a more clear case is shown in Fig.1a for the brown dwarf candidate GD 165B discovered by Becklin \& Zuckerman (1988): The spectra observed by Jones et al.(1994) can best be explained by the predicted spectrum by our dusty model (solid line) but not at all by our dust-free model (dashed line). Further evidence for dust in late $\mathrm{M}$ dwarfs is also found in the weakening of $\mathrm{TiO}$ bands (Jones \& Tsuji 1997). Somewhat ironically, however, our dusty models could not explain the spectra of the genuine brown dwarf Gl 229B discovered by Nakajima et al.(1995) just when we have completed our dusty models, although our earlier model without dust (Tsuji \& Ohnaka 1995) could (Tsuji et al 1996b).

In the $(I-J, J-K)$ diagram shown in Fig.1b, $J-K$ of $\mathrm{M}$ dwarfs (Leggett 1992) remains nearly constant up to $\mathrm{M} 6 \mathrm{~V}$ but shows reddening at $\mathrm{M} 7 \mathrm{~V}$ and later including GD $165 \mathrm{~B}$ (Tinney et al. 1993). This trend is well explained by our dusty models (solid line) but not by dust-free models (dashed line). Recently, three brown dwarf candidates, one of which (DBD J1228-1547) is confirmed to be brown dwarf, have been found by the DENIS survey (Delfosse et al. 1997), and they appear in Fig.1b near GD 165B which we already know to be well dusty. Thus, the agreement between the observed colours of late $\mathrm{M}$ dwarfs (and brown dwarf candidates) and the predicted 

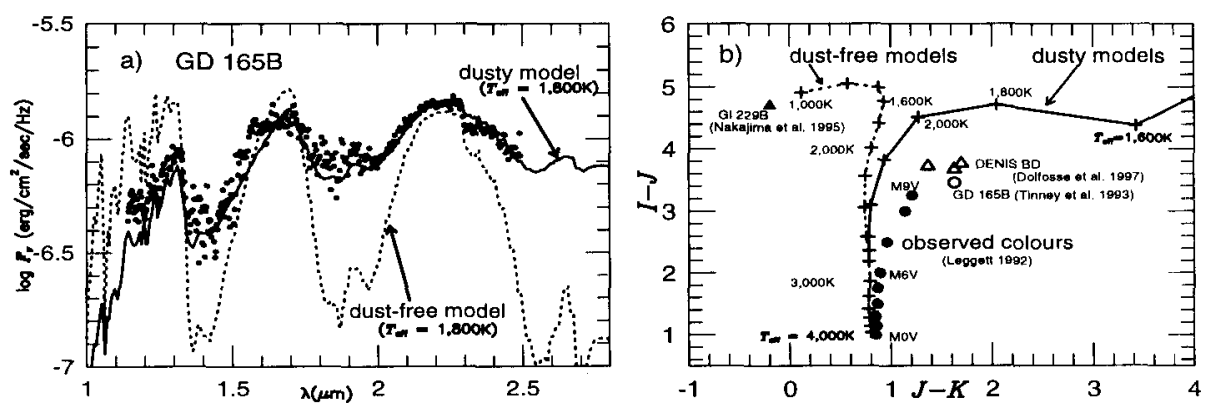

Figure 1. a) The spectrum of GD 165B (dots: Jones et al. 1994) is compared with the predictions by dusty (solid line) and dust-free (dashed line) models $\left(T_{\mathrm{eff}}=1,800 \mathrm{~K}\right.$ ), and shows definite evidence for dust in this brown dwarf candidate. b) Predicted loci based on dusty (solid line) and non-dusty (dashed line) models on the $(I-J, J-K)$ diagram. Observed colours are shown for M dwarfs (filled circles), GD 165B (open circle), DENIS brown dwarf candidates (open triangles), and GL $229 \mathrm{~B}$ (filled triangle). Note that dusty and non-dusty cases can clearly be separated.

ones based on our dusty models can be regarded as evidence for dust in most late $\mathrm{M}$ dwarfs and brown dwarf candidates. On the other hand, the predicted locus based on our dust-free models turns to the blue at about $T_{\text {eff }}=1,600 \mathrm{~K}$ and explains the observed colous of Gl $229 \mathrm{~B}$ quite well. Thus there should be two kinds of brown dwarfs which can clearly be separeted on $(I-J, J-\bar{K})$ diagram: blue brown dwarfs of non-dusty type (e.g. Gl 229B) and red brown dwarfs of dusty type (e.g. DBD J1228-1547). However, M dwarfs later than about M7V are probably all dusty and red.

\section{Discussion and Conclusions: how dust forms in stellar photosphere}

The presence of dust in the photospheres of M dwarfs later than M7V and hot brown dwarfs can be regarded as well established. The fact that the majority of VLMOs are dusty implies that dust exists in the photospheres of these objects throughout their lifetime (or as long as the Hubble time in the case of $\mathrm{M}$ dwarfs). This should be possible if dust formation and destruction are repeating forever, that is the dust is in detailed balance with the gas. This means that the dust-gas mixture is in LTE, and our assumption that dust forms in LTE (Sect.2) is justified by the very fact that dust is present in a large number of VLMOs.

The case of the very cool brown dwarf Gl 229B is clearly different. Probably grain size turns to be larger than the critical radius, and dust may begin to grow (that is an irreversible process starts). Then, the dust can no longer be in detailed balance with the gas and will finally segregate from the gas (or dust clouds will form). Volatile gas will then appear in the observable layer in the photosphere and this case explains the observation of Gl 229B (Tsuji et al. 1996b). Thus, the presence of two kinds of brown dwarfs (Sect.3) can be explained. However, where and why the transition from the dust-gas detailed balance regime to dust-gas segregation phase occurs is unknown. Probably, some mechanism to prevent dust-growth is working until a certain phase. Certainly, more work is needed to fully understand the spectra of $M$ dwarfs and brown dwarfs, and to better determine their basic properties. For this purpose, dust is again an important ingredient, since the Universe is everywhere dusty and $M$ dwarfs are no exception.

\section{References}

Becklin, E. E. and Zuckerman, B. (1988) Nature, 336, 656

Delfosse, X. et al. (1997) A\&A, in press

Jones, H. R. A., Longmore, A. J., Jameson, R. F., Mountain, C. M. (1994) MNRAS, 267, 413

Jones, H. R. A. and Tsuji, T. (1997) ApJL, 480, L39

Leggett, S.K. (1992) ApJS, 82, 351

Nakajima, T., Oppenheimer, B. R., Kulkarni, S. R., Goljmowski, D. A., Matthews, K. and Durrance, S. T. (1995) Nature, 378, 464

Tinney, C. G., Mould, J. R. and Ried, I. N. (1993) AJ, 105, 1045

Tsuji, T. and Ohnaka, K. (1995) in Elementary Processes in Dense Plasmas eds. S. Ichimaru and S. Ogata, ArldisonWesley, p.193

Tsuji, T., Ohnaka, K. and Aoki, W. (1996a) A\&A, 305, L1

Tsuji, T., Ohnaka, K., Aoki, W. and Nakajima, T. (1996b) A\&A, 308, L29 